\title{
Existence of solutions to boundary value problems for a higher-dimensional difference system
}

\author{
Tao Zhou ${ }^{a}$, Xia Liub,c,*, Haiping Shid \\ a School of Business Administration, South China University of Technology, Guangzhou 510640, China. \\ ${ }^{b}$ Oriental Science and Technology College, Hunan Agricultural University, Changsha 410128, China. \\ 'Science College, Hunan Agricultural University, Changsha 410128, China. \\ ${ }^{d}$ Modern Business and Management Department, Guangdong Construction Polytechnic, Guangzhou 510440, China. \\ Communicated by C. Zaharia
}

\begin{abstract}
By using critical point theory, some new criteria are obtained for the existence of a nontrivial homoclinic orbit to a higher order difference system containing both many advances and retardations. The proof is based on the Mountain Pass Lemma in combination with periodic approximations. Related results in the literature are generalized and improved. (C)2017 All rights reserved.
\end{abstract}

Keywords: Boundary value problems, higher-dimensional, mountain pass lemma, critical point theory. 2010 MSC: 39A10, 47J30, 58E05.

\section{Introduction}

Difference equations, the discrete analogs of differential equations, have been applied as models in vast areas such as finance insurance, biological populations, disease control, genetic study, physical field, and computer application technology, etc. Because of their importance, many literature and monographs deal with its existence and uniqueness problems. For example, see [1-7, 12-20, 22, 24-27].

We denote by $\mathbb{N}, \mathbb{Z}$ and $\mathbb{R}$ the sets of all natural numbers, integers and real numbers respectively. Throughout this paper, for all $a, b \in \mathbb{Z}$, we define $\mathbb{Z}(a)=\{a, a+1, \cdots\}, \mathbb{Z}(a, b)=\{a, a+1, \cdots, b\}$ when $a \leqslant b$. Let the symbol * denote the transpose of a vector. In the following and in the sequel, for any $\mathrm{n} \in \mathbb{N},|\cdot|$ will denote the Euclidean norm in $\mathbb{R}^{n}$, defined by

$$
|X|=\left(\sum_{i=1}^{n} X_{i}^{2}\right)^{\frac{1}{2}}, \quad \forall X=\left(X_{1}, X_{2}, \cdots, X_{n}\right) \in \mathbb{R}^{n} .
$$

\footnotetext{
*Corresponding author

Email addresses: zhoutaoscut@hotmail.com (Tao Zhou), xia991002@163.com (Xia Liu), shp7971@163. com (Haiping Shi)
} doi:10.22436/jnsa.010.10.37 
Consider the nonlinear higher order difference system

$$
\sum_{i=0}^{n} r_{i}\left(X_{k-i}+X_{k+i}\right)=f\left(k, X_{k+\Gamma}, \cdots, X_{k}, \cdots, X_{k-\Gamma}\right), \quad n \in \mathbb{N}, \quad k \in \mathbb{Z}(1, T),
$$

with boundary value conditions

$$
X_{1-\tau}=X_{2-\tau}=\cdots=X_{0}=0, \quad X_{T+1}=X_{T+2}=\cdots=X_{T+\tau}=0,
$$

where $r_{i}$ is a real number, $\Gamma$ is a given nonnegative integer, $T$ and $m$ are given positive integers,

$$
f=\left(f_{1}, f_{2}, \cdots, f_{m}\right)^{*} \in C\left(\mathbb{R}^{2 \Gamma+2} \times \mathbb{R}^{m}, \mathbb{R}\right),
$$

$\tau=\max \{n, \Gamma\}, f \in C\left(\mathbb{R}^{2 \Gamma+2}, \mathbb{R}\right)$.

We may regard (1.1) as being a discrete analogue of the following $2 n$-th order functional differential equation

$$
\left[r(t) X^{(n)}\right]^{(n)}=f(t, X(t+\Gamma), \cdots, X(t), \cdots, X(t-\Gamma)), \quad t \in \mathbb{R} .
$$

When $\mathrm{m}=1, \mathrm{n}=1, \Gamma=1$, Smets and Willem [28] proved the existence of solitary waves with prescribed speed on infinite lattices of particles with nearest neighbor interaction for the following forward and backward differential difference equation

$$
c^{2} u^{\prime \prime}(t)=V^{\prime}(u(t+1)-u(t))-V^{\prime}(u(t)-u(t-1)), \quad t \in \mathbb{R} .
$$

Equations similar in structure to (1.3) arise in the study of the existence of periodic solutions and homoclinic orbits for functional differential equations, see [8-11].

In 2007, Cai and Yu [2] established some criteria for the existence of periodic solutions of a $2 n$-th order difference equation

$$
\Delta^{n}\left(r_{k-n} \Delta^{n} u_{k-n}\right)+f\left(k, u_{k}\right)=0, \quad n \in \mathbb{Z}(3), \quad k \in \mathbb{Z},
$$

by using the Linking Theorem.

If $\Gamma=0, \mathrm{Hu}$ and Huang in 2008 [12] applied the critical point theorem to prove the existence of periodic solution of a higher order difference equation as the following type

$$
\sum_{i=0}^{n} r_{i}\left(X_{k-i}+X_{k+i}\right)+f\left(k, X_{k}\right)=0, \quad n \in \mathbb{N}, \quad k \in \mathbb{Z} .
$$

Chen and Tang [3] in 2011 obtained some new existence criteria to guarantee the $2 n$-th order nonlinear difference equation

$$
\Delta^{n}\left(r_{k-n} \Delta^{n} u_{k-n}\right)+q_{k} u_{k}=f\left(k, u_{k+n}, \cdots, u_{k}, \cdots, u_{k-n}\right), \quad n \in \mathbb{Z}(3), \quad k \in \mathbb{Z},
$$

has at least one or infinitely many homoclinic orbits by establishing a proper variational framework and using the critical point theory.

By using the critical point theory, Deng and Shi [6] in 2010 obtained some sufficient conditions for the existence and multiplicity of the boundary value problems to a class of second order functional difference equations

$$
L u_{k}=f\left(k, u_{k+1}, u_{k}, u_{k-1}\right),
$$

with boundary value conditions

$$
\Delta \mathrm{u}_{0}=\mathrm{A}, \quad \mathrm{u}_{\mathrm{T}+1}=\mathrm{B} .
$$

Recently, Liu et al. [20] studied the following $2 n$-th order nonlinear difference equation

$$
\Delta^{n}\left(\gamma_{i-n+1} \Delta^{n} u_{i-n}\right)=(-1)^{n} f\left(i, u_{i+1}, u_{i}, u_{i-1}\right), \quad n \in \mathbb{Z}(1), \quad i \in \mathbb{Z}(1, T),
$$


with boundary value conditions

$$
\Delta \mathfrak{u}_{1-n}=\Delta \mathfrak{u}_{2-n}=\cdots=\Delta \mathfrak{u}_{0}=0, \quad \mathfrak{u}_{k+1}=\mathfrak{u}_{k+2}=\cdots=\mathfrak{u}_{k+n}=0,
$$

and gave some new results of solutions for the mixed boundary value problem by using the Mountain Pass Lemma.

Motivated by the above papers [6,20], the intention of this paper is to consider the boundary value problem (1.1) with (1.2) in a more general sense. More exactly our results represent the extensions to higher-dimensional difference systems containing both many advances and retardations. We establish some new sufficient conditions ensuring the existence of solutions to boundary value problems for such a system. One of our results generalizes an existing result. In fact, one can see the following Remark 1.4 for details.

Throughout the paper, for a function $F$, we let $F_{i}^{\prime}\left(Y_{1}, \cdots, Y_{i} \cdots, Y_{n}\right)$ denote the partial derivative of $F$ on the $i$ variable.

For basic knowledge of variational methods, the reader is referred to [21, 23].

Our main results are the following theorems.

Theorem 1.1. Assume that $\mathrm{F}$ satisfies the following assumptions:

$\left(\mathrm{F}_{1}\right)$ there exists a function $\mathrm{F}\left(\mathrm{t}, \mathrm{Y}_{\Gamma}, \cdots, \mathrm{Y}_{0}\right) \in \mathrm{C}^{1}\left(\mathbb{R}^{\Gamma+2} \times \mathbb{R}^{\mathrm{m}}, \mathbb{R}\right)$ such that

$$
\sum_{i=-\Gamma}^{0} F_{2+\Gamma+i}^{\prime}\left(t+i, Y_{\Gamma+i}, \cdots, Y_{i}\right)=f\left(t, Y_{\Gamma}, \cdots, Y_{0}, \cdots, Y_{-\Gamma}\right)
$$

$\left(\mathrm{F}_{2}\right)$ there exist constants $\mathrm{c}_{1}>0, \mathrm{c}_{2}>0$ and $\alpha>2$ such that

$$
\mathrm{F}\left(\mathrm{t}, \mathrm{Y}_{\Gamma}, \cdots, \mathrm{Y}_{0}\right) \geqslant \mathrm{c}_{1}\left(\sqrt{\sum_{j=0}^{\Gamma} \mathrm{Y}_{j}^{2}}\right)^{\alpha}-\mathrm{c}_{2}, \quad \forall\left(\mathrm{t}, \mathrm{T}_{\Gamma}, \cdots, \mathrm{Y}_{0}\right) \in \mathbb{R}^{\Gamma+2} .
$$

Then the boundary value problem (1.1) with (1.2) possesses at least one solution.

Remark 1.2. The results of Theorem 1.1 ensure that the boundary value problem (1.1) with (1.2) possesses at least one solution. However, in some cases, we are interested in the existence of nontrivial solutions.

Theorem 1.3. Assume that $\mathrm{r}$ and $\mathrm{F}$ satisfy $\left(\mathrm{F}_{1}\right),\left(\mathrm{F}_{2}\right)$ and the following assumptions:

$\left(r_{1}\right) r_{0}+\sum_{s=1}^{n}\left|r_{s}\right|<0$;

$\left(\mathrm{F}_{3}\right)$ for all $\left(\mathrm{t}, \mathrm{T}_{\Gamma}, \cdots, \mathrm{Y}_{0}\right) \in \mathbb{R}^{\Gamma+2}$,

$$
\lim _{\rho \rightarrow 0} \frac{F\left(t, Y_{\Gamma}, \cdots, Y_{0}\right)}{\rho^{2}}=0, \quad \rho=\sqrt{\sum_{j=0}^{\Gamma} Y_{j}^{2}} .
$$

Then the boundary value problem (1.1) with (1.2) possesses at least two nontrivial solutions.

Remark 1.4. Theorem 1.3 generalizes [27, Theorem 1.2] which is the special case of our Theorem 1.3 by letting $\mathrm{m}=1$ and $\Gamma=1$.

Theorem 1.5. Assume that $\mathrm{r}$ and $\mathrm{F}$ satisfy $\left(\mathrm{F}_{1}\right)$ and the following assumptions are satisfied:

$$
\left(\mathrm{r}_{2}\right)-\mathrm{r}_{0}+\sum_{s=1}^{\mathrm{n}}\left|\mathrm{r}_{\mathrm{s}}\right| \leqslant 0
$$


$\left(\mathrm{F}_{4}\right) \mathrm{Y}_{0} \mathrm{f}\left(\mathrm{t}, \mathrm{Y}_{\Gamma}, \cdots, \mathrm{Y}_{0}, \cdots, \mathrm{Y}_{-\Gamma}\right)>0$, for $\mathrm{Y}_{0} \neq 0$, for all $\mathrm{t} \in \mathbb{R}$.

Then the boundary value problem (1.1) with (1.2) has no nontrivial solution.

This paper is divided into five parts. First of all, Section 2 presents variational structure. The second, in Section 3, we shall recall some related fundamental results and present some lemmas. Then, Section 4 is dedicated to the proof of main results. Finally, in Section 5, we shall give two examples to illustrate the applicability of the main results.

\section{Variational structure}

Our main tool is the critical point theory. We shall establish the corresponding variational framework for the boundary value problem (1.1) with (1.2). We start by some basic notations for the reader's convenience.

Let $\mathbb{R}^{\mathrm{mT}}$ be the real Euclidean space with dimension $m T . \mathbb{R}^{\mathrm{mT}}$ can be equipped with the inner product $\langle X, Y\rangle$ and norm $\|X\|$ as follows

$$
\langle X, Y\rangle:=\sum_{j=1}^{T} X_{j} \cdot Y_{j}, \quad \forall X, Y \in \mathbb{R}^{m T},
$$

and

$$
\|X\|:=\left(\sum_{j=1}^{T}\left|X_{j}\right|^{2}\right)^{\frac{1}{2}}, \quad \forall X \in \mathbb{R}^{\mathrm{mT}},
$$

where $|\cdot|$ denotes the Euclidean norm in $\mathbb{R}^{m}$, and $X_{j} \cdot Y_{j}$ denotes the usual scalar product in $\mathbb{R}^{m}$.

On the other hand, we define the norm $\|\cdot\|_{s}$ on $\mathbb{R}^{\mathrm{mT}}$ as follows

$$
\|X\|_{s}=\left(\sum_{j=1}^{T}\left|X_{j}\right|^{s}\right)^{\frac{1}{s}}
$$

for all $X \in \mathbb{R}^{\mathrm{m} T}$ and $s>1$.

Since $\|X\|_{s}$ and $\|X\|_{2}$ are equivalent, there exist constants $K_{1}, K_{2}$ such that $K_{2} \geqslant K_{1}>0$, and

$$
\mathrm{K}_{1}\|\mathrm{X}\|_{2} \leqslant\|\mathrm{X}\|_{s} \leqslant \mathrm{~K}_{2}\|\mathrm{X}\|_{2}, \quad \forall \mathrm{X} \in \mathbb{R}^{\mathrm{mT}} .
$$

For all $X \in \mathbb{R}^{\mathrm{mT}}$, define the functional $\mathrm{J}$ on $\mathbb{R}^{\mathrm{mT}}$ as follows:

$$
J(X):=\frac{1}{2} \sum_{k=1}^{T} \sum_{i=0}^{n} r_{i}\left(X_{k-i}+X_{k+i}\right) X_{k}-\sum_{k=1}^{T} F\left(k, X_{k+\Gamma}, \cdots, X_{k}\right) .
$$

Since $X_{1-\tau}=X_{2-\tau}=\cdots=X_{0}=0, X_{T+1}=X_{T+2}=\cdots=X_{T+\tau}=0$, then

$$
\frac{\partial J(X)}{\partial X_{k, l}}=\sum_{i=0}^{n} r_{i}\left(X_{k-i, l}+X_{k+i, l}\right)-f_{l}\left(k, X_{k+\Gamma}, \cdots, X_{k}, \cdots, X_{k-\Gamma}\right), \quad l \in \mathbb{Z}(1, m), \quad k \in \mathbb{Z}(1, T) .
$$

Therefore, $X \in \mathbb{R}^{\mathrm{m} T}$ is a critical point of $J$, i.e., $\mathrm{J}^{\prime}(X)=0$ if and only if

$$
\sum_{i=0}^{n} r_{i}\left(X_{k-i, l}+X_{k+i, l}\right)-f_{l}\left(k, X_{k+\Gamma}, \cdots, X_{k}, \cdots, X_{k-\Gamma}\right)=0, \quad l \in \mathbb{Z}(1, m), k \in \mathbb{Z}(1, T) .
$$


That is,

$$
\sum_{i=0}^{n} r_{i}\left(X_{k-i}+X_{k+i}\right)-f\left(k, X_{k+\Gamma}, \cdots, x_{k}, \cdots, x_{k-\Gamma}\right)=0, \quad k \in \mathbb{Z}(1, T) .
$$

Thus, we reduce the problem of finding boundary value problem (1.1) with (1.2) to that of seeking critical points of the functional $\mathrm{J}$ in $\mathbb{R}^{\mathrm{mT}}$.

For all $X \in \mathbb{R}^{\mathrm{mT}}$, J can be rewritten as

$$
J(X)=-\frac{1}{2}\langle D M X, M X\rangle-\sum_{k=1}^{T} F\left(k, X_{k+\Gamma}, \cdots, X_{k}\right),
$$

where $X=\left\{X_{k}\right\} \in E_{T}, X_{k}=\left(X_{k, 1}, X_{k, 2}, \cdots, X_{k, m}\right)^{*}, k \in \mathbb{Z}(1, T)$, and

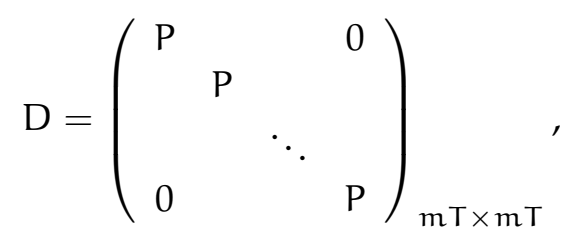

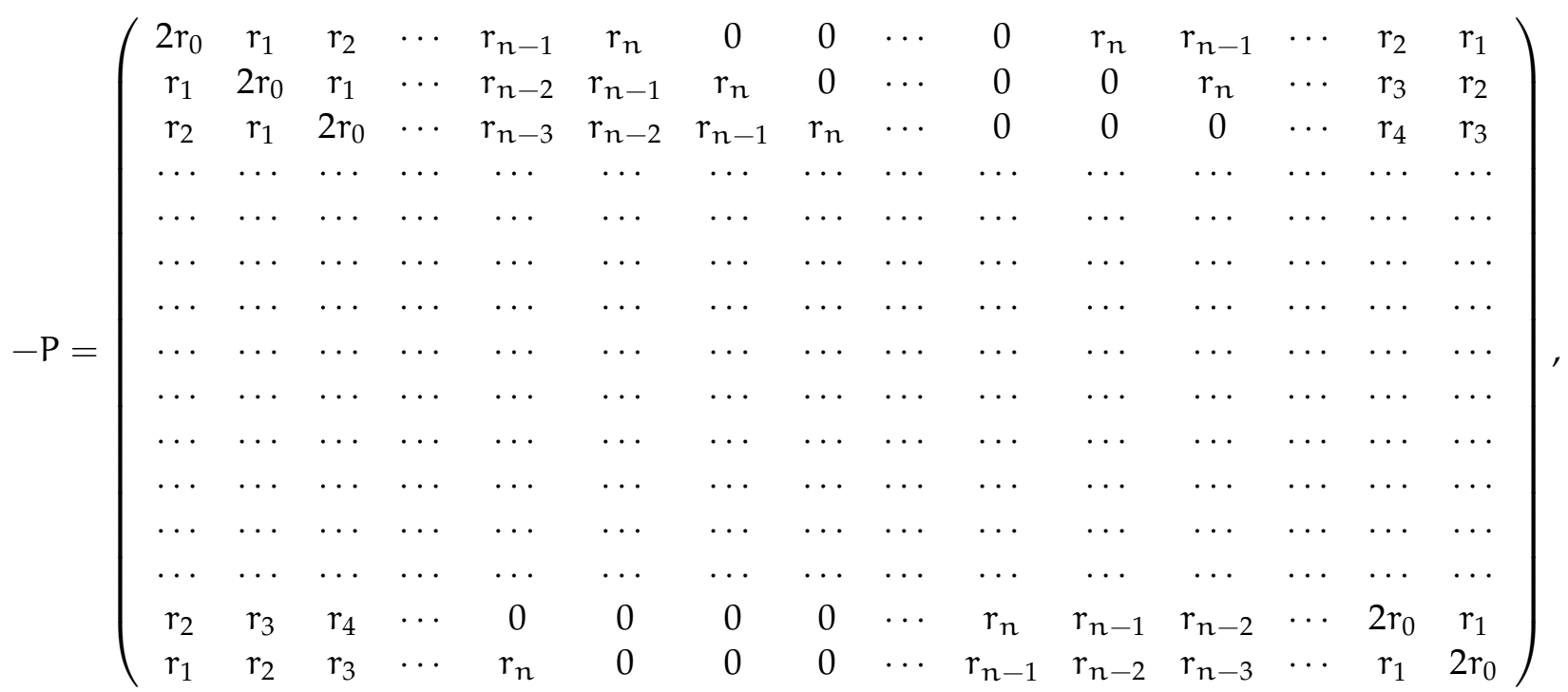

is a $T \times T$ matrix. Assume that the eigenvalues of $P$ are $\lambda_{1}, \lambda_{2}, \cdots, \lambda_{T}$ respectively. It is easy to see that $P$ is a circulant matrix [29] denoted by

$$
P:=\operatorname{Circ}\left\{-2 r_{0},-r_{1},-r_{2}, \cdots,-r_{n}, 0, \cdots, 0,-r_{n},-r_{n-1}, \cdots,-r_{2},-r_{1}\right\}
$$

By [29], the eigenvalues of $P$ are

$$
\lambda_{j}=-2 r_{0}-\sum_{s=1}^{n} r_{s}\left\{\exp i \frac{2 j \pi}{T}\right\}^{s}-\sum_{s=1}^{n} r_{s}\left\{\exp i \frac{2 j \pi}{T}\right\}^{T-s}=-2 \sum_{s=0}^{n} r_{s} \cos \left(\frac{2 j s \pi}{T}\right),
$$

where $j=1,2, \cdots, T$.

By (2.2), we have

$$
-2 r_{0}-2 \sum_{s=1}^{n}\left|r_{s}\right| \leqslant \lambda_{j} \leqslant-2 r_{0}+2 \sum_{s=1}^{n}\left|r_{s}\right|, \quad j=1,2, \cdots, T
$$

\section{Main lemmas}

In order to apply critical point theory to study the existence of boundary value problem (1.1) with (1.2), we shall state some lemmas, which will be used in the proofs of our main results. 
Let $E$ be a real Banach space, $J \in C^{1}(E, \mathbb{R})$, i.e., $J$ is a continuously Fréchet-differentiable functional defined on E. J is said to satisfy the Palais-Smale condition (P.S. condition for short) if any sequence $\left\{X^{(n)}\right\}_{n \in \mathbb{N}} \subset E$ for which $\left\{J\left(X^{(n)}\right)\right\}_{n \in \mathbb{N}}$ is bounded and $J^{\prime}\left(X^{(n)}\right) \rightarrow 0(n \rightarrow \infty)$ possesses a convergent subsequence in $E$.

Let $B_{\rho}$ denote the open ball in $E$ about 0 of radius $\rho$ and let $\partial B_{\rho}$ denote its boundary.

Lemma 3.1 (Mountain Pass Lemma [21, 23]). Let $\mathrm{E}$ be a real Banach space and $\mathrm{J} \in \mathrm{C}^{1}(\mathrm{E}, \boldsymbol{R})$ satisfy the P.S. condition. If $\mathrm{J}(0)=0$ and

$\left(\mathrm{J}_{1}\right)$ there exist constants $\rho, \alpha>0$ such that $\mathrm{J}_{\partial_{\mathrm{B}}} \geqslant \alpha$; and

$\left(\mathrm{J}_{2}\right)$ there exists $\mathrm{e} \in \mathrm{E} \backslash \mathrm{B}_{\rho}$ such that $\mathrm{J}(\mathrm{e}) \leqslant 0$.

Then J possesses a critical value $\mathrm{c} \geqslant \alpha$ given by

$$
c=\inf _{g \in \Upsilon} \max _{s \in[0,1]} J(g(s)),
$$

where

$$
\Upsilon=\{\mathrm{g} \in \mathrm{C}([0,1], \mathrm{E}) \mid \mathrm{g}(0)=0, \mathrm{~g}(1)=\mathrm{e}\} .
$$

Lemma 3.2. Assume that $\left(\mathrm{r}_{1}\right)$ and $\left(\mathrm{F}_{1}\right)-\left(\mathrm{F}_{3}\right)$ are satisfied. Then $\mathrm{J}$ satisfies the P.S. condition.

Proof. It follows from $\left(\mathrm{r}_{1}\right)$ that $\mathrm{P}$ is positive definite. Denote

$$
\lambda_{\max }=\max \left\{\lambda_{j} \mid j=1,2, \cdots, T\right\} .
$$

Let $\left\{X^{(n)}\right\}_{n \in \mathbb{N}} \subset \mathbb{R}^{m T}$ be such that $\left\{J\left(X^{(n)}\right)\right\}_{n \in \mathbb{N}}$ is bounded and $J^{\prime}\left(X^{(n)}\right) \rightarrow 0$ as $n \rightarrow \infty$. By $\left(F_{2}\right)$, (2.1), (2.2) and (3.1), we have

$$
\begin{aligned}
J\left(X^{(n)}\right) & =-\frac{1}{2}\left\langle D M X^{(n)}, M X^{(n)}\right\rangle-\sum_{k=1}^{T} F\left(k, X_{k+\Gamma}^{(n)}, \cdots, X_{k}^{(n)}\right) \\
& \leqslant \frac{1}{2} \lambda_{\max }\left\|X^{(n)}\right\|^{2}-c_{1} \sum_{k=1}^{T}\left[\sqrt{\left.\sum_{j=0}^{\Gamma}\left(X_{k+j}^{(n)}\right)^{2}\right]^{\alpha}+c_{2} T}\right. \\
& \leqslant \frac{1}{2} \lambda_{\max }\left\|X^{(n)}\right\|^{2}-c_{1} K_{1}^{\alpha}\left\|X^{(n)}\right\|^{\alpha}+c_{2} T .
\end{aligned}
$$

Since $J\left(X^{(n)}\right)$ is anti-coercive and $\alpha>2$, then the P.S. condition follows immediately.

\section{Proof of the main results}

Now, we shall finish proof of our main results by using the variational method and critical point theory.

Proof of Theorem 1.1. For any $X=\left(X_{1}, X_{2}, \cdots, X_{T}\right)^{*} \in \mathbb{R}^{m T}$, we have

$$
\begin{aligned}
J(X) & =-\frac{1}{2}\langle D M X, M X\rangle-\sum_{k=1}^{T} F\left(k, X_{k+\Gamma}, \cdots, X_{k}\right) \\
& \leqslant \frac{1}{2} \lambda_{\max }\|X\|^{2}-c_{1} \sum_{k=1}^{T}\left[\sqrt{\sum_{j=1}^{\Gamma} X_{k+j}^{2}}\right]^{\alpha}+c_{2} T \\
& \leqslant \frac{1}{2} \lambda_{\max }\|X\|^{2}-c_{1} K_{1}^{\alpha}\|X\|^{\alpha}+c_{2} T \rightarrow-\infty,
\end{aligned}
$$


as $\|X\| \rightarrow+\infty$. Due to the continuity of $J(X)$, the above inequality implies that there exist upper bounds of values of functional $J$. Classical calculus shows that $J$ attains its maximal value at some point which is just the critical point of $J$ and the result follows.

Proof of Theorem 1.3. By $\left(F_{3}\right)$, for any $\varepsilon=\frac{1}{4(\Gamma+2)} \lambda_{\min }\left(\lambda_{\min }\right.$ can be referred to (3.1)), there exists $\delta>0$, such that

$$
\left|F\left(k, Y_{\Gamma}, \cdots, Y_{0}\right)\right| \leqslant \frac{1}{4(\Gamma+2)} \lambda_{\min } \sum_{j=0}^{\Gamma} Y_{j}^{2}, \quad \forall k \in \mathbb{Z}(1, T),
$$

for $\sqrt{\sum_{j=0}^{\Gamma} Y_{j}^{2}} \leqslant \sqrt{\Gamma+2} \delta$.

For any $X=\left(X_{1}, X_{2}, \cdots, X_{T}\right)^{*} \in \mathbb{R}^{m T}$ and $\|X\| \leqslant \delta$, we have $\left|X_{k}\right| \leqslant \delta, k \in \mathbb{Z}(1, T)$. Then,

$$
\begin{aligned}
J(X) & =-\frac{1}{2}\langle D M X, M X\rangle-\sum_{k=1}^{T} F\left(k, X_{k+\Gamma}, \cdots, X_{k}\right) \\
& \geqslant \frac{1}{2} \lambda_{\min }\|X\|^{2}-\frac{1}{4(\Gamma+2)} \lambda_{\min } \sum_{k=1}^{T} \sum_{j=0}^{\Gamma} X_{k+j}^{2} \\
& \geqslant \frac{1}{2} \lambda_{\min }\|X\|^{2}-\frac{1}{4} \lambda_{\min }\|X\|^{2} \\
& =\frac{1}{4} \lambda_{\min }\|X\|^{2} .
\end{aligned}
$$

Take $a \triangleq \frac{1}{4} \lambda_{\min } \delta^{2}>0$. Therefore, $J(X) \geqslant a>0$, for all $X \in \partial B_{\delta}$. At the same time, we have also proved that there exist constants $a>0$ and $\delta>0$ such that $\left.J\right|_{\partial B_{\delta}} \geqslant a$. That is to say J satisfies the condition $\left(J_{1}\right)$ of the Mountain Pass Lemma.

The rest of the proof is similar to that of [27], but for the sake of completeness, we give the details.

For our setting, clearly $\mathrm{J}(0)=0$. In order to exploit the Mountain Pass Lemma in critical point theory, we need to verify other conditions of the Mountain Pass Lemma. By Lemma 3.2, J satisfies the P.S. condition. So it suffices to verify the condition $\left(\mathrm{J}_{2}\right)$.

From the proof of the P.S. condition, we know $J(X) \leqslant \frac{1}{2} \lambda_{\max }\|X\|^{2}-c_{1} K_{1}^{\alpha}\|X\|^{\alpha}+c_{2} T$. Since $\alpha>2$, we can choose $\bar{X}$ large enough to ensure that $J(\bar{X})<0$. By the Mountain Pass Lemma, J possesses a critical value $c \geqslant a>0$, where $c=\inf _{g \in \Upsilon} \sup _{s \in[0,1]} J(g(\theta))$ and $\Upsilon=\left\{g \in C\left([0,1], \mathbb{R}^{m T}\right) \mid g(0)=0, g(1)=\bar{X}\right\}$.

Let $\tilde{X} \in \mathbb{R}^{m \top}$ be a critical point associated to the critical value $c$ of $J$, i.e., $J(\tilde{X})=c$. Similar to the proof of the P.S. condition, we know that there exists $\hat{X} \in \mathbb{R}^{\mathrm{mT}}$ such that $\mathrm{J}(\hat{\mathrm{X}})=\mathrm{c}_{\max }=\max _{\theta \in[0,1]} \mathrm{J}(\mathrm{g}(\theta))$.

If $\tilde{X} \neq \hat{X}$, then the conclusion of Theorem 1.3 holds. Otherwise, $\tilde{X}=\hat{X}$. Then we get that all points on the critical level are solutions. Otherwise we have two of them. $\hat{X}$ must be nontrivial since the value is greater than the critical value which is positive.

Proof of Theorem 1.5. By matrix theory, combining with $\left(\mathrm{r}_{2}\right)$, we have that the eigenvalues of $\mathrm{P}$ are nonpositive, i.e., $\lambda_{j} \leqslant 0$ for all $j \in \mathbb{Z}(1, T)$. For the sake of contradiction, we assume that the boundary value problem (1.1) with (1.2) has a nontrivial solution. Then, J has a nonzero critical point $X^{\star}$. Since

$$
\frac{\partial J}{\partial X_{k}^{\star}}=\sum_{i=0}^{n} r_{i}\left(X_{k-i}^{\star}+X_{k+i}^{\star}\right)-f\left(k, X_{k+\Gamma}^{\star}, \cdots, X_{k}^{\star}, \cdots, X_{k-\Gamma}^{\star}\right),
$$

we get

$$
\sum_{k=1}^{T} f\left(k, X_{k+\Gamma}^{\star}, \cdots, X_{k}^{\star}, \cdots, X_{k-\Gamma}^{\star}\right) X_{k}^{\star}=\sum_{k=1}^{T} \sum_{i=0}^{n} r_{i}\left(X_{k-i}^{\star}+X_{k+i}^{\star}\right) X_{k}^{\star}=-\left\langle D M X^{\star}, M X^{\star}\right\rangle \leqslant 0 .
$$


On the other hand, it follows from $\left(\mathrm{F}_{4}\right)$ that

$$
\sum_{i=1}^{T} f\left(k, X_{k+\Gamma}^{\star}, \cdots, X_{k}^{\star}, \cdots, X_{k}^{\star}-\Gamma\right) X_{k}^{\star}>0 .
$$

This contradicts (4.1) and hence the proof is finished.

\section{Examples}

As an application of Theorems 1.3 and 1.5, we give two examples to illustrate our main results.

Example 5.1. For $n \in \mathbb{N}, k \in \mathbb{Z}(1, T)$, let

$$
f\left(k, X_{k+\Gamma}, \cdots, X_{k}, \cdots, X_{k-\Gamma}\right)=\alpha X_{k} \sum_{j=0}^{\Gamma}(k-j)\left(\sum_{i=0}^{\Gamma} X_{k+i-j}^{2}\right)^{\frac{\alpha}{2}-1} .
$$

It is easy to verify all the assumptions of Theorem 1.3 are satisfied. Consequently, two nontrivial solutions are obtained.

Example 5.2. For $n \in \mathbb{N}, k \in \mathbb{Z}(1, T)$, let

$$
f\left(k, X_{k+\Gamma}, \cdots, x_{k}, \cdots, X_{k-\Gamma}\right)=12 X_{k} \sum_{j=0}^{\Gamma}(k-j)\left(\sum_{i=0}^{\Gamma} x_{k+i-j}^{6}\right)^{5} .
$$

It is easy to verify all the assumptions of Theorem 1.5 are satisfied. Consequently, there is no nontrivial solution.

\section{Acknowledgment}

This project is supported by the National Natural Science Foundation of China (No. 11501194) and Department of Education of Guangdong Province for Excellent Young College Teacher of Guangdong Province.

\section{References}

[1] R. P. Agarwal, Difference equations and inequalities, Theory, methods, and applications, Second edition, Monographs and Textbooks in Pure and Applied Mathematics, Marcel Dekker, Inc., New York, (2000). 1

[2] X.-C. Cai, J.-S. Yu, Existence of periodic solutions for a 2nth-order nonlinear difference equation, J. Math. Anal. Appl., 329 (2007), 870-878. 1

[3] P. Chen, X.-H. Tang, Existence of homoclinic orbits for 2 nth-order nonlinear difference equations containing both many advances and retardations, J. Math. Anal. Appl., 381 (2011), 485-505. 1

[4] P. Cull, M. Flahive, R. Robson, Difference equations, From rabbits to chaos, Undergraduate Texts in Mathematics, Springer, New York, (2005).

[5] X.-Q. Deng, Nonexistence and existence results for a class of fourth-order difference mixed boundary value problems, J. Appl. Math. Comput., 45 (2014), 1-14.

[6] X.-Q. Deng, H.-P. Shi, On boundary value problems for second order nonlinear functional difference equations, Acta Appl. Math., 110 (2010), 1277-1287. 1

[7] X.-Q. Deng, H.-P. Shi, X.-L. Xie, Periodic solutions of second order discrete Hamiltonian systems with potential indefinite in sign, Appl. Math. Comput., 218 (2011), 148-156. 1

[8] C.-J. Guo, D. O'Regan, Y.-T. Xu, R. P. Agarwal, Existence of subharmonic solutions and homoclinic orbits for a class of even higher order differential equations, Appl. Anal., 90 (2011), 1169-1183. 1

[9] C.-J. Guo, D. O’Regan, Y.-T. Xu, R. P. Agarwal, Existence and multiplicity of homoclinic orbits of a second-order differential difference equation via variational methods, Appl. Math. Inform. Mech., 4 (2012), 1-15. 
[10] C.-J. Guo, D. O'Regan, Y.-T. Xu, R. P. Agarwal, Existence of homoclinic orbits for a class of first-order differential difference equations, Acta Math. Sci. Ser. B Engl. Ed., 35 (2015), 1077-1094.

[11] C.-J. Guo, Y.-T. Xu, Existence of periodic solutions for a class of second order differential equation with deviating argument, J. Appl. Math. Comput., 28 (2008), 425-433. 1

[12] R.-H. Hu, L.-H. Huang, Existence of periodic solutions of a higher order difference system, J. Korean Math. Soc., 45 (2008), 405-423. 1, 1

[13] M. Jia, Standing waves for discrete nonlinear Schrödinger equations, Electron. J. Differential Equations, 2016 (2016), 9 pages.

[14] J.-H. Leng, Existence of periodic solutions for higher-order nonlinear difference equations, Electron. J. Differential Equations, 2016 (2016), 10 pages.

[15] J.-H. Leng, Periodic and subharmonic solutions for 2 nth-order $\phi_{c}$-Laplacian difference equations containing both advance and retardation, Indag. Math. (N.S.), 27 (2016), 902-913.

[16] X. Liu, H.-P. Shi, Y.-B. Zhang, Nonexistence and existence of solutions for a fourth-order discrete mixed boundary value problem, Proc. Indian Acad. Sci. Math. Sci., 124 (2014), 179-191.

[17] X. Liu, H.-P. Shi, Y.-B. Zhang, On the nonexistence and existence of solutions for a fourth-order discrete Dirichlet boundary value problem, Quaest. Math., 38 (2015), 203-216.

[18] X. Liu, Y.-B. Zhang, H.-P. Shi, Existence and nonexistence results for a $2 \mathrm{n}$-th order $\mathrm{p}$-Laplacian discrete Dirichlet boundary value problem, translated from Izv. Nats. Akad. Nauk Armenii Mat., 49 (2014), 133-143, J. Contemp. Math. Anal., 49 (2014), 287-295.

[19] X. Liu, Y.-B. Zhang, H.-P. Shi, Existence and nonexistence results for a fourth-order discrete Neumann boundary value problem, Studia Sci. Math. Hungar., 51 (2014), 186-200.

[20] X. Liu, Y.-B. Zhang, H.-P. Shi, Nonexistence and existence results for a 2 nth-order discrete mixed boundary value problem, Rev. R. Acad. Cienc. Exactas Fís. Nat. Ser. A Math. RACSAM, 109 (2015), 303-314. 1, 1

[21] J. Mawhin, M. Willem, Critical point theory and Hamiltonian systems, Applied Mathematical Sciences, SpringerVerlag, New York, (1989). 1, 3.1

[22] G. Molica Bisci, D. Repovš, Existence of solutions for p-Laplacian discrete equations, Appl. Math. Comput., 242 (2014), 454-461. 1

[23] P. H. Rabinowitz, Minimax methods in critical point theory with applications to differential equations, CBMS Regional Conference Series in Mathematics, Published for the Conference Board of the Mathematical Sciences, Washington, DC; by the American Mathematical Society, Providence, RI, (1986). 1, 3.1

[24] H. Sedaghat, Nonlinear difference equations, Theory with applications to social science models, Mathematical Modelling: Theory and Applications, Kluwer Academic Publishers, Dordrecht, (2003). 1

[25] H.-P. Shi, Boundary value problems of second order nonlinear functional difference equations, J. Difference Equ. Appl., 16 (2010), 1121-1130.

[26] H.-P. Shi, Z.-Z. Liu, Z.-G. Wang, Dirichlet boundary value problems for second order p-Laplacian difference equations, Rend. Istit. Mat. Univ. Trieste, 42 (2010), 19-29.

[27] H.-P. Shi, X. Liu, Y.-B. Zhang, Nonexistence and existence results for a 2 nth-order discrete Dirichlet boundary value problem, Kodai Math. J., 37 (2014), 492-505. 1, 1.4, 4

[28] D. Smets, M. Willem, Solitary waves with prescribed speed on infinite lattices J. Funct. Anal., 149 (1997), 266-275. 1

[29] R. R. Stoll, Linear algebra and matrix theory, McGraw-Hill Book Company, Inc., New York-Toronto-London, (1952). 2 